\title{
Downlink Multiuser Hybrid Beamforming for MmWave Massive MIMO-NOMA System with Imperfect CSI
}

\author{
Jing Jiang $\mathbb{D}$, Ming Lei $\mathbb{D}^{\mathbb{D}}$, and Huanhuan Hou \\ Shaanxi Key Laboratory of Information Communication Network and Security, Xian University of Posts and Telecommunications, \\ Xian 710121, China \\ Correspondence should be addressed to Ming Lei; leiming19940812@163.com
}

Received 29 August 2018; Revised 1 March 2019; Accepted 28 April 2019; Published 13 May 2019

Guest Editor: Khalil Sayidmarie

Copyright (c) 2019 Jing Jiang et al. This is an open access article distributed under the Creative Commons Attribution License, which permits unrestricted use, distribution, and reproduction in any medium, provided the original work is properly cited.

\begin{abstract}
This paper aims to provide a comprehensive scheme with limited feedback for downlink millimeter wave (mmWave) multiuser multiple-input multiple-output (MIMO) nonorthogonal multiple access (NOMA) system. Based on the feedback of the best beam and the channel quality information (CQI) on this beam, the users are grouped into a cluster having the same or coherent best beam and the maximal CQI-difference. To further reduce the intercluster interference, only the candidate cluster can join the cluster set whose intercluster correlation with the existing clusters is lower than threshold. Based on the results of clustering, mmWave hybrid beamforming is designed. To improve the user experience, each cluster selects the best beam of the user with the higher guaranteed rate requirement as the analog beamforming vector. For digital beamforming, the weak user applies the block diagonalization algorithm based on the strong user's effective channel to reduce its intracluster interference. Finally, an intracluster power allocation algorithm is developed to maximize the power difference in each cluster which is beneficial to improve the successive interference cancelation (SIC) performance of the strong user. Finally, simulation results show that the proposed MIMO-NOMA scheme offers a higher sum rate than the traditional orthogonal multiple access (OMA) scheme under practical conditions.
\end{abstract}

\section{Introduction}

In contrast to the conventional orthogonal multiple access (OMA), nonorthogonal multiple access (NOMA) allows different users to efficiently share the same resources (i.e., time, frequency, space, and code) at different power levels. Exploiting their respective channel gain differences, multiuser signals are separated at receivers by successive interference cancelation (SIC). Thus, it is an important technique for $5 \mathrm{G}$ systems which can significantly enhance system capacity and overall spectral efficiency [1-3].

NOMA can flexibly combine other $5 \mathrm{G}$ technologies, e.g., MIMO, cognitive radio, cooperative communication, and channel coding [3]. Because MIMO-NOMA systems can significantly increase the number of supportable users and in turn improve the system sum capacity, the research of MIMO-NOMA has been carried out widely [4-7]. Multiple users with distinct channel gains are grouped into MIMONOMA clusters. The users in the same cluster are scheduled on NOMA basis. The intracluster interference is mitigated by SIC based on the obvious channel gain difference. Multiple clusters may utilize the multiuser MIMO principles to cancel the intercluster interference. References [4-8] prove that NOMA combined with MIMO techniques could achieve great system performance gains over OMA.

Thanks to the abundant bandwidth resources, millimeter wave (mmWave) frequency becomes a natural choice to achieve Gigabit data rates $[9,10]$. The number of supportable users can be more than the number of radio frequency $(\mathrm{RF})$ chains at the same resources by exploiting MIMO-NOMA in mmWave system. On the other hand, the channel of mmWave is highly directional. For mmWave MIMO-NOMA system, the users in a same cluster use the same beam and the different clusters use the distinct different beams. For the users in a same cluster, the obvious power difference in a same beam could assure good SIC performance. For different clusters, the distinct different beams are beneficial to greatly reduce the intercluster inference $[11,12]$. Therefore, 
mmWave MIMO-NOMA can effectively improve the spectral efficiency and cope with the demands of massive connectivity.

Considering mmWave MIMO-NOMA system, [11] analyzes the achievable sum rate of the proposed beamspace MIMO-NOMA in a typical mmWave channel mode, which shows an obvious performance gain compared with the existing beamspace MIMO. Zero-forcing beamforming (ZF$\mathrm{BF})$ is designed to reduce the interbeam interference in the beamspace. Furthermore, a dynamic power allocation is proposed to maximize the achievable sum rate which contains the intrabeam and interbeam power optimization. Reference [12] proposes a hybrid analog/digital beamforming scheme with a power allocation algorithm aiming to maximize the energy efficiency. Users having a high channel correlation and large channel gain difference are grouped as a cluster. Reference [13] investigates the maximization of the sum rate of the system with clusters of 2-user or more-user. It decomposes the original problem into power and beam gain allocation problems. Reference [14] proposes a hybrid analog/digital beamforming framework to maximize the sum rate and analyzes the effect of beam misalignment on rate performance.

Most of the existing works on mmWave MIMO-NOMA, such as [11-14], have assumed perfect knowledge of channel state information (CSI) at the transmitter, which is difficult to realize in practice. Reference [15] proposes a hybrid precoding scheme based on one-stage feedback, which makes use of all feedback overhead to enable precise beam information and takes advantage of the second-order channel statistics to mitigate multiuser interference. Reference [16] proposes the random beamforming in which base station (BS) broadcasts the random beams and the users with the enough signal strength feed their channel quality information (CQI) back to the BS.

MmWave MIMO-NOMA system with limited feedback has been studied but with limited results. Reference [15] has not yet considered user clustering and the intracluster interference cancelation with limited feedback. Reference [16] reduces the feedback overhead, but the channel measurement is time-consuming and complicated. In this paper, we put forward a comprehensive scheme with imperfect CSI for mmWave MIMO-NOMA system. To reduce the hardware complexity, we apply low RF chains structure at the BS, where the hybrid analog/digital beamforming is considered. The major contributions of this paper can be listed as follows:

(1) The user clustering is designed based on the limited feedback scheme. References [16-21] put forward some feedback designs for MIMO-NOMA, e.g., on bit feedback, the path loss information, and other second-order statistics. Considering mmWave user could acquire the big gain in the specific beam, one-bit feedback or the path loss information is not accurate enough. In this paper, the limited feedback uses the best beam and CQI value based on this beam. The users having the same or adjacent best beam are sorted based on their CQI values, and the users with the maximal CQI-difference are formed a cluster. To further reduce the intercluster interference, the user clustering algorithm applies the intercluster correlation threshold to assure only the cluster with the distinct different beam can join the cluster set. Numerical results prove that it acquires obvious sum rate improvement by adequately exploiting the high directional feature of mmWave.

(2) We propose a novel hybrid analog/digital beamforming algorithm. In general, the users in a same cluster will use the beam of the strong user as the beamforming vector $[11,12]$. The beamforming gains are assured to achieve first for the strong users. However, the weak users would require higher data rate. To improve the quality of user experience and the user fairness, for each cluster, we select the beam of the user with the higher rate requirement as the analog beamforming vector. For digital beamforming, the prior work [12] applies ZF-BF to cancel the intercluster interference. For mmWave communication, the distinct different beams cause very little interference as to the sparsity of mmWave channel. In this paper, the digital beamforming is designed for the strong user and the weak user, respectively. The strong user utilizes the singular value decomposition (SVD) of its effective channel to acquire more gains from digital beamforming. Especially, the weak user exploits the block diagonalization (BD) algorithm to mitigate the intracluster interference from the strong user in the same cluster.

(3) As the power difference between the two users in a same cluster increases, not only is the SIC performance of the strong user improved, but also the intracluster interference of the weak user is reduced. So we derive an intracluster power allocation scheme that maximizes the power difference under the users' quality of service (QoS) requirements. We derive a closed-form optimal power allocation for the two users in a cluster by deriving the exact bounds for the power allocation coefficient region.

(4) Finally, we evaluate the sum rate performance of the downlink MIMO-NOMA system using the proposed beamforming, user clustering and power allocation algorithms. The simulations are done with a wide range of beam correlation thresholds, including the interuser and intercluster thresholds in the user clustering algorithm. Both of the perfect CSI and random selection cases are considered in the simulations. Numerical results also compare the sum rate performances of MIMO-OMA and the proposed MIMO-NOMA and illustrate the significance of the proposed MIMO-NOMA scheme.

The rest of this paper is organized as follows. Section 2 presents the system and channel model of mmWave MIMONOMA system. Sections 3, 4, and 5 describe the designs of user clustering, hybrid beamforming and power allocation, respectively. The simulation configuration and results are presented and discussed in Section 6, and finally, Section 7 concludes this paper.

Notation. In the reminder of this paper, we use the following notations: $(\cdot)^{T}$ and $(\cdot)^{\mathrm{H}}$ denote the transpose and Hermitian transpose, respectively, $\|\cdot\|$ denotes the Frobenius norm, and $\mathscr{C} \mathcal{N}(a, b)$ denotes the distribution of circularly symmetric complex Gaussian random variable with mean $a$ and covariance $b$. 


\section{System and Channel Model}

Consider a downlink mmWave beamforming NOMA transmission system, in which one BS communicates with $L$ clusters. Each cluster consists of multiple single-antenna users. The BS is equipped with $N_{\mathrm{TX}}$ antennas and $N_{\mathrm{RF}}\left(N_{\mathrm{TX}} \geq N_{\mathrm{RF}}\right)$ $\mathrm{RF}$ chains for reducing the hardware complexity. We consider the fully connected architecture that each RF chain is linked to all antennas by using multiple phase shifters. We assume that the number of clusters served by the BS is equal to that of $\mathrm{RF}$ chains $\left(L=N_{\mathrm{RF}}\right)$. Although each cluster can contain more than two users, we suppose here that there are two users in each cluster for simplicity. This is consistent with the standard implementation of NOMA in long term evolution advanced (LTE-A) as well [22].

On the downlink, the BS applies an $N_{\mathrm{RF}} \times 2 L$ baseband beamforming $\mathbf{W}=\left[\mathbf{w}_{1,1}, \mathbf{w}_{1,2}, \ldots, \mathbf{w}_{L, 1}, \mathbf{w}_{L, 2}\right]$ followed by an $N_{\mathrm{TX}} \times N_{\mathrm{RF}}$ RF beamforming $\mathbf{F}_{\mathrm{RF}}=\left[\mathbf{f}_{1}^{\mathrm{RF}}, \mathbf{f}_{2}^{\mathrm{RF}}, \ldots, \mathbf{f}_{N_{\mathrm{RF}}}^{\mathrm{RF}}\right]$. The sampled transmitted signal is therefore

$$
\mathbf{x}=\mathbf{F}_{\mathrm{RF}} \mathbf{W},
$$

where $\mathbf{s}=\left[\sqrt{P_{1} \alpha_{1}} s_{1,1}, \sqrt{P_{1}\left(1-\alpha_{1}\right)} s_{1,2}, \ldots, \sqrt{P_{L} \alpha_{L}} s_{L, 1}\right.$, $\left.\sqrt{P_{L}\left(1-\alpha_{L}\right)} s_{L, 2}\right]^{T}$ is the $2 L \times 1$ vector of transmitted symbols in which $s_{l, 1}$ and $s_{l, 2}$ denote the signals for the 1-th and 2-th in the $l$-th cluster, respectively. Similarly, $0<\alpha_{l}<1$ and $1-\alpha_{l}$ stand for the power allocation coefficients for the two users in the $l$-th cluster. $P_{l}$ is the power allocated to the $l$-th cluster.

The received signal of the $i$-th user in the $l$-th cluster can be represented as follows:

$$
\begin{aligned}
y_{l, i} & =\mathbf{h}_{l, i} \mathbf{x}+n_{l, i} \\
= & \mathbf{h}_{l, i} \mathbf{F}_{\mathrm{RF}}\left(\mathbf{w}_{l, 1} \sqrt{P_{l} \alpha_{l}} s_{l, 1}+\mathbf{w}_{l, 2} \sqrt{P_{l}\left(1-\alpha_{l}\right)} s_{l, 2}\right) \\
& +\mathbf{h}_{l, i} \mathbf{F}_{\mathrm{RF}} \sum_{j \neq l}^{L}\left(\mathbf{w}_{j, 1} \sqrt{P_{j} \alpha_{j}} s_{j, 1}+\mathbf{w}_{j, 2} \sqrt{P_{j}\left(1-\alpha_{j}\right)} s_{j, 2}\right) \\
& +n_{l, i},
\end{aligned}
$$

where $\mathbf{h}_{l, i}$ is the $1 \times N_{\text {TX }}$ vector that represents the mmWave channel of the $i$-th user in the $l$-th cluster. $n_{l, i} \sim \mathscr{C} \mathcal{N}\left(0, \sigma^{2}\right)$ denotes additive white complex Gaussian noise. Moreover, the second term is the intercluster interference from other clusters. Without loss of generality, for each cluster, we assume that $\left\|\mathbf{h}_{l, 1} \mathbf{F}_{\mathrm{RF}} \mathbf{w}_{l, 1}\right\| \geq\left\|\mathbf{h}_{l, 2} \mathbf{F}_{\mathrm{RF}} \mathbf{w}_{l, 2}\right\|$ where $1 \leq l \leq L$. Following this, the 1-th and 2-th are defined as the strong user and weak user in each cluster, respectively.

Due to the limited scattering in mmWave channel, we adopt a well-established geometric channel model with $\mathrm{P}$ scatterers $[23,24]$. We assume that each scatterer contributes a single propagation path from the BS to user. Then, the channel $\mathbf{h}_{l, i}(i \in\{1,2\}, l \in\{1, \ldots, L\})$ can be expressed as

$$
\mathbf{h}_{l, i}=\sqrt{N_{\mathrm{TX}}} \frac{\beta_{l, i}^{0} \mathbf{a}\left(\theta_{l, i}^{0}\right)^{\mathrm{H}}}{\sqrt{1+d_{l, i}^{\eta_{\mathrm{LOS}}}}}+\sqrt{N_{\mathrm{TX}}} \sum_{f=1}^{\mathrm{P}} \frac{\beta_{l, i}^{f} \mathbf{a}\left(\theta_{l, i}^{f}\right)^{\mathrm{H}}}{\sqrt{1+d_{l, i}^{\eta_{\mathrm{NLOS}}}}},
$$

where the first and second terms denote the line-of-sight (LoS) component and non-line-of-sight (NLoS) components of the $i$-th user in the $l$-th cluster, respectively. $d_{l, i}$ denotes the distance from the BS to the $i$-th user in the $l$-th cluster. $\eta_{L O S}$ and $\eta_{N L O S}$ are the path loss exponents corresponding to the LoS and NLoS paths, respectively. $\beta_{l, i}^{f} \in \mathscr{C} \mathcal{N}(0,1)$ where $0 \leq f \leq \mathrm{P}$ denotes the complex gain of the $f$-th path between the BS and the $i$-th user in the $l$-th cluster. $\theta_{l, i}^{f} \in[-1,1]$ is the $f$-th path's normalized direction, whereas $\mathbf{a}\left(\theta_{l, i}^{f}\right)$ is the antenna array steering vector with respect to $\theta_{l, i}^{f}$. If we assume a uniform linear array (ULA) is used at the BS, $\mathbf{a}\left(\theta_{l, i}^{f}\right)$ can be written as [25]

$$
\mathbf{a}\left(\theta_{l, i}^{f}\right)=\frac{1}{\sqrt{N_{\mathrm{TX}}}}\left[1, e^{-j \pi \theta_{l, i}^{f}}, \ldots, e^{-j \pi\left(N_{\mathrm{TX}}-1\right) \theta_{l, i}^{f}}\right]^{T} .
$$

where $\theta_{l, i}^{f} \in[-1,1]$ is related to the angle of departure $\vartheta \in[-\pi / 2, \pi / 2]$ as $\theta_{l, i}^{f}=(2 D / \lambda) \sin (\vartheta)$ [26]. $D$ denotes the distance between antenna elements and $\lambda$ denotes the signal wavelength satisfying $D=2 / \lambda$ at mmWave frequencies.

\section{User Clustering}

Appropriately selecting two users which are served in a cluster can help improve the performance of NOMA multiuser beamforming. On the one hand, the big difference of the channel gains can improve SIC performance of the users with high channel gains and reduce the intracluster interference of the users with low channel gains. On the other hand, the beamforming vector is shared by all users in the same cluster. If the channels of those users in a same cluster are highly correlated, the beamforming vectors can acquire the definite array gains and properly cancel the intercluster interference from other clusters. Reference [4] proposed a clustering algorithm selecting user-pair having a high channel correlation and large channel gain difference as set in the same cluster, which requires full CSI. However, the full CSI feedback to the BS is not feasible in practice due to prohibitively high feedback overhead. So we propose a user clustering algorithm with limited feedback information, which only requires the indexes of the best beams and the channel quality information (CQI) values.

The index of the best beam and the user's CQI are measured and sent back as follows. In the first stage, the BS broadcasts pilot signals on the every vector in the beamforming codebook. In the second stage, each user chooses the best beam and measures the CQI with this beamforming vector. At last, the user sends the index of the best beam and CQI to the BS. The best beam of the $i$-th user $\widehat{\mathbf{b}}_{i}$ can be selected according to the following criterion as [27]

$$
\widehat{\mathbf{b}}_{i}=\underset{\mathbf{b}_{i} \in \Phi}{\arg \max }\left|\mathbf{h}_{i} \mathbf{b}_{i}\right|^{2},
$$

where $\Phi$ represents the beamforming codebook with size $|\Phi|=2^{B_{\mathrm{RF}}}$ (i.e., $B_{\mathrm{RF}}$ feedback bits) and consists of the steering vectors $\mathbf{a}\left(-1+2 k_{\mathrm{Q}} / 2^{B_{\mathrm{RF}}}\right), k_{\mathrm{Q}}=0,1, \ldots, 2^{B_{\mathrm{RF}}}-1$. They have 
the same form of the array response vector in (4). $\mathbf{b}_{i}$ is the beamforming vector selected from $\Phi$.

As discussed in $[24,28]$, the effect of LoS component is dominant in mmWave channels, compared to those of NLoS components. Therefore, the above mmWave channel model in (3) can be simplified as

$$
\mathbf{h}_{l, i}=\sqrt{N_{\mathrm{TX}}} \frac{\beta_{l, i} \mathbf{a}\left(\theta_{l, i}\right)^{\mathrm{H}}}{\sqrt{1+d_{l, i}^{\eta}}},
$$

where the subscript 0 of the LoS component is omitted for the simplification of notation. Then, the effective channel gain of the user $i$ on the beam $\mathbf{b}_{i}$ in (5) can be expressed as

$$
\begin{aligned}
\left|\mathbf{h}_{i} \mathbf{b}_{i}\right|^{2} & =\frac{N_{\mathrm{TX}}\left|\beta_{i}\right|^{2}\left|\mathbf{a}\left(\theta_{i}\right)^{\mathrm{H}} \mathbf{a}\left(\varphi_{i}\right)\right|^{2}}{1+d_{l, i}^{\eta}} \\
& =\frac{\left|\beta_{i}\right|^{2}\left|\sum_{l=0}^{N_{\mathrm{TX}}-1} e^{-j \pi l\left(\theta_{i}-\varphi_{i}\right)}\right|^{2}}{N_{\mathrm{TX}}\left(1+d_{l, i}^{\eta}\right)} \\
& =\frac{\left|\beta_{i}\right|^{2} \sin ^{2}\left(\pi N_{\mathrm{TX}}\left(\theta_{i}-\varphi_{i}\right) / 2\right)}{N_{\mathrm{TX}}\left(1+d_{l, i}^{\eta}\right) \sin ^{2}\left(\pi\left(\theta_{i}-\varphi_{i}\right) / 2\right)} \\
& =\frac{\left|\beta_{i}\right|^{2}}{1+d_{l, i}^{\eta}} F_{N_{\mathrm{TX}}}\left(\pi\left[\theta_{i}-\varphi_{i}\right]\right)
\end{aligned}
$$

where $\varphi_{i}$ is the predefined normalized direction of $\mathbf{b}_{i}$. $F_{N_{\mathrm{TX}}}(x)$ is the Fejér kernel, which goes to zero quickly when $x$ increases. Therefore, based on the criterion in (5), the normalized direction $\widehat{\varphi}_{i}$ of the selected beam $\widehat{\mathbf{b}}_{i}$ has the least difference from $\theta_{i}$ in the codebook. This means that the normalized direction of each user can be approximately the normalized direction of its selected beam. The correlation of the selected beam between user $i$ and user $j$ can be rewritten as

$$
\begin{aligned}
\rho_{(i, j)} & =\frac{\left|\widehat{\mathbf{b}}_{i}^{\mathrm{H}} \widehat{\mathbf{b}}_{j}\right|}{\left\|\widehat{\mathbf{b}}_{i}\right\|\left\|\widehat{\mathbf{b}}_{j}\right\|}=\frac{\left|\mathbf{a}\left(\widehat{\varphi}_{i}\right)^{\mathrm{H}} \mathbf{a}\left(\widehat{\varphi}_{j}\right)\right|}{\left\|\mathbf{a}\left(\widehat{\varphi}_{i}\right)\right\|\left\|\mathbf{a}\left(\widehat{\varphi}_{j}\right)\right\|} \\
& =\frac{\left|\sum_{l=0}^{N_{\mathrm{TX}}-1} e^{-j \pi l\left(\widehat{\varphi}_{i}-\widehat{\varphi}_{j}\right)}\right|}{N_{\mathrm{TX}}}=\sqrt{\frac{F_{N_{\mathrm{TX}}}\left(\pi\left[\widehat{\varphi}_{i}-\widehat{\varphi}_{j}\right]\right)}{N_{\mathrm{TX}}}} .
\end{aligned}
$$

Based on the above beam correlation expression in (8) and channel expression in (6), the channel correlation between users $i$ and user $j$ can be simplified as

$$
\begin{aligned}
\operatorname{Corrh}_{(i, j)} & =\frac{\left|\mathbf{h}_{i} \mathbf{h}_{j}^{\mathrm{H}}\right|}{\left\|\mathbf{h}_{i}\right\|\left\|\mathbf{h}_{j}\right\|}=\frac{\left|\mathbf{a}\left(\theta_{i}\right)^{\mathrm{H}} \mathbf{a}\left(\theta_{j}\right)\right|}{N_{\mathrm{TX}}} \\
& =\frac{\left|\sum_{l=0}^{N_{\mathrm{TX}}-1} e^{-j \pi l\left(\theta_{i}-\theta_{j}\right)}\right|}{N_{\mathrm{TX}}}
\end{aligned}
$$

$$
\begin{aligned}
& =\sqrt{\frac{F_{N_{\mathrm{TX}}}\left(\pi\left[\theta_{i}-\theta_{j}\right]\right)}{N_{\mathrm{TX}}}} \\
& \stackrel{(a)}{\approx} \sqrt{\frac{F_{N_{\mathrm{TX}}\left(\pi\left[\widehat{\varphi}_{i}-\widehat{\varphi}_{j}\right]\right)}^{N_{\mathrm{TX}}}}{\|}}=\frac{\left|\widehat{\mathbf{b}}_{i}^{\mathrm{H}} \widehat{\mathbf{b}}_{j}\right|}{\left\|\widehat{\mathbf{b}}_{i}\right\|\left\|\widehat{\mathbf{b}}_{j}\right\|}=\rho_{(i, j)},
\end{aligned}
$$

where $\operatorname{Corrh}_{(i, j)}$ denotes the channel correlation between user $i$ and user $j$. (a) follows from that $\theta_{i}$ and $\theta_{j}$ are approximately $\widehat{\varphi}_{i}$ and $\widehat{\varphi}_{j}$ based on the above analysis, respectively. So the correlation between the users' channels $\operatorname{Corrh}_{(i, j)}$ can be replaced with the correlation between their selected beams $\rho_{(i, j)}$.

CQI is an indicator carrying information about the channel condition. In LTE, considering a single BS system, the received SNR for each user $i$ can be written as [29]

$$
\kappa_{i}=\frac{P_{\mathrm{t}}\left\|\mathbf{h}_{i} \widehat{\mathbf{b}}_{i}\right\|^{2}}{\sigma^{2}},
$$

where $P_{\mathrm{t}}$ is the transmit power and $\sigma^{2}$ is the power of received noise and interference. The measured SNR $\kappa_{i}$ is then mapped to a discrete CQI value $g_{i}$ using a chipset vendor specific mapping table [30]. Because the positive correlation between channel gain and CQI value, we will just calculate the difference of CQI values between users instead of their channel gain difference.

It is proved in [8] that the more users are admitted to a cluster, the lower is the achieved sum rate, which illustrates the tradeoff between the sum rate and maximum number of admitted users. Considering the tradeoff, we assume that each cluster admits 2 users. Based on the above analysis, a limited feedback downlink MIMO-NOMA user clustering algorithm is described in Algorithm 1, in which the number of clusters $L=N_{R F}$ and each cluster contains 2 users.

Algorithm 1 can be summarized as follows. In the first step, the BS calculates the beam correlation of all possible user-pairs and puts the user-pair having a beam correlation higher than the threshold $\mu$ into set $T$. In the second step, during each loop, the BS searches for a new user-pair $\left(i^{*}, j^{*}\right)$ having the maximum CQI value difference from $T$. Then the BS calculates the beam correlations between the user $i^{*}$ and the selected strong users in set A. If all the correlations are lower than the threshold $\zeta$, then the user-pair $\left(i^{*}, j^{*}\right)$ is added to a new cluster and the user $i^{*}$ is put into A. Otherwise, no users are added. The loop is repeated consecutively until $L$ user clusters are selected.

\section{Two-Stage Hybrid Beamforming}

4.1. The Optimization of Analog Beamforming. For analog beamforming, one analog beamforming vector is designed for one user in traditional design [31], while two users in a same cluster should share one analog beamforming vector in MIMO-NOMA. Furthermore, considering the fairness of QoS, the rate requirement of the weak user may be greater than that of the strong user in a cluster. So we select the 


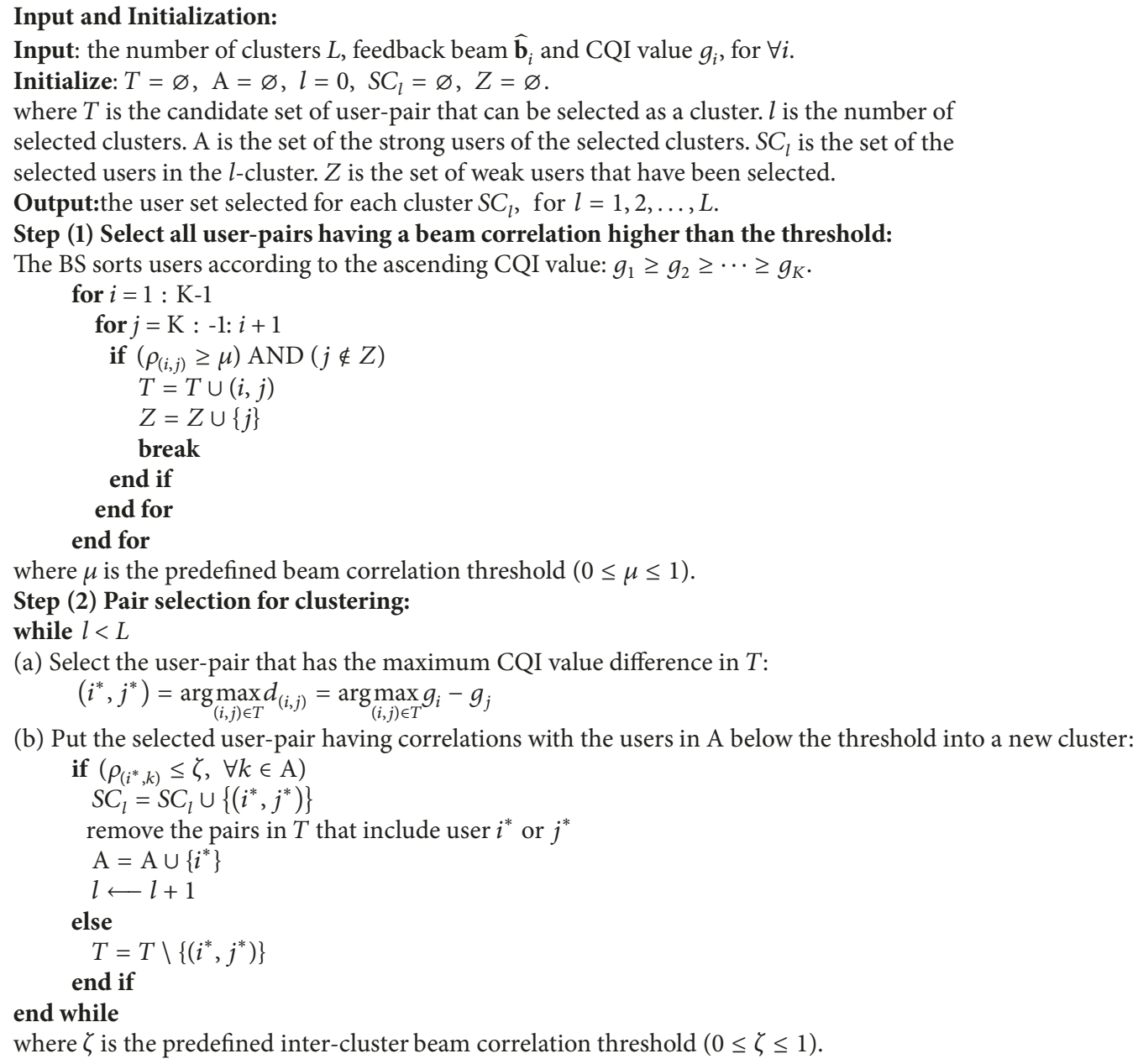

(b) Put the selected user-pair having correlations with the users in A below the threshold into a new cluster:

Algorithm 1: User clustering with beam and CQI.

feedback beam of the user with the maximum guaranteed rate requirement as the analog beamforming vector in each cluster, i.e., $\widehat{i}_{l}=\arg \max _{i \in S C_{l}} \widetilde{R}_{l, i}$, where $\widetilde{R}_{l, i}$ is the guaranteed rate requirement of the $i$-user in the $l$-cluster.

Based on the above analysis, we can design the analog beamforming vector for the $l$-th cluster according to the following criterion:

$$
\mathbf{f}_{l}^{\mathrm{RF}}=\widehat{\mathrm{b}}_{\hat{i}_{l}} .
$$

Then, the analog beamforming matrix can be obtained as $\mathbf{F}_{\mathrm{RF}}=\left[\mathbf{f}_{1}^{\mathrm{RF}}, \mathbf{f}_{2}^{\mathrm{RF}}, \ldots, \mathbf{f}_{N_{\mathrm{RF}}}^{\mathrm{RF}}\right]$.

4.2. The Design of Digital Beamforming. In this subsection we design the digital beamforming. After the analog beamforming, the BS trains the effective channels with users as

$$
\widetilde{\mathbf{h}}_{l, i}=\mathbf{h}_{l, i} \mathbf{F}_{\mathrm{RF}}, \quad \text { for } l=1, \ldots, L \text { and } i=1,2 \text {. }
$$

The dimension of the effective channel $\widetilde{\mathbf{h}}_{l, i}$ is $1 \times N_{\mathrm{RF}}$ which is much less than that of its original channel $\mathbf{h}_{l, i}$.
This is different from the algorithms proposed in $[32,33]$ in which the effective channel has a larger $1 \times N_{T X}$ dimension. Then, each user quantizes its effective channel utilizing a random vector quantization (RVQ) codebook $\eta$ of size $2^{B_{E C}}$ and feeds the index of the vector $\widehat{\mathbf{h}}_{l, i}$ back to the BS with $B_{E C}$ bits, where $\widehat{\mathbf{h}}_{l, i}=\operatorname{argmax}_{\widehat{\mathbf{h}}_{l, i} \in \eta}\left\|\widetilde{\mathbf{h}}_{l, i} \widehat{\mathbf{h}}_{l, i}^{\mathrm{H}}\right\|$ [34]. Finally, the BS designs its digital beamforming based on the quantized channels.

The user clustering and analog beamforming mitigate the intercluster interference. Then we can design the digital beamforming vector for the weak user to reduce its intracluster interference. We take the design of the digital beamforming vector $\mathbf{w}_{l, 2}$ for the weak user in the $l$-th cluster as an example. First, we construct a complementary matrix $\overline{\mathbf{H}}_{l, 2}$ that contains the other user in the same cluster, i.e., $\overline{\mathbf{H}}_{l, 2}=$ $\widehat{\mathbf{h}}_{l, 1}$. Note that $\overline{\mathbf{H}}_{l, 2} \in \mathbb{C}^{1 \times N_{\mathrm{RF}}}$ and $N_{\mathrm{RF}} \geq 2$. It is obviously $\operatorname{rank}\left(\overline{\mathbf{H}}_{l, i}\right)=1$. Then, we define the SVD of $\overline{\mathbf{H}}_{l, 2}$ as

$$
\overline{\mathbf{H}}_{l, 2}=\overline{\mathbf{U}}_{l, 2} \bar{\Sigma}_{l, 2}\left[\overline{\mathbf{V}}_{l, 2}^{(1)} \overline{\mathbf{V}}_{l, 2}^{(0)}\right]^{\mathrm{H}}
$$


where $\overline{\mathbf{V}}_{l, 2}^{(0)}$ holds the last $\left(N_{\mathrm{RF}}-1\right)$ right singular vectors and forms an orthogonal basis for the null space of $\overline{\mathbf{H}}_{l, 2}$. We perform SVD on $\widehat{\mathbf{h}}_{l, 2} \overline{\mathbf{V}}_{l, 2}^{(0)}$ as

$$
\widehat{\mathbf{h}}_{l, 2} \overline{\mathbf{V}}_{l, 2}^{(0)}=\widetilde{\mathbf{U}}_{l, 2} \widetilde{\Sigma}_{l, 2} \widetilde{\mathbf{V}}_{l, 2}
$$

Finally, we can design the digital beamforming vector $\mathbf{w}_{l, 2}$ as

$$
\mathbf{w}_{l, 2}=\frac{\overline{\mathbf{V}}_{l, 2}^{(0)} \widetilde{\mathbf{V}}_{l, 2}^{(1)}}{\left\|\mathbf{F}_{R F} \overline{\mathbf{V}}_{l, 2}^{(0)} \widetilde{\mathbf{V}}_{l, 2}^{(1)}\right\|}, \quad \text { for } l=1, \ldots, L,
$$

where $\widetilde{\mathbf{V}}_{l, 2}^{(1)}$ denotes the first column of $\widetilde{\mathbf{V}}_{l, 2}$. For the strong user, we can design a digital beamforming vector to increase its capacity and in turn reduce its interuser interference. More exactly, we define the SVD of $\widehat{\mathbf{h}}_{l, 1}$ as

$$
\widehat{\mathbf{h}}_{l, 1}=\mathbf{U}_{l, 1} \Sigma_{l, 1} \mathbf{V}_{l, 1}^{\mathrm{H}} \text {. }
$$

Then we can design the digital beamforming vector $\mathbf{w}_{l, 1}$ for the strong user as

$$
\mathbf{w}_{l, 1}=\frac{\mathbf{V}_{l, 1}^{(1)}}{\left\|\mathbf{F}_{R F} \mathbf{V}_{l, 1}^{(1)}\right\|}, \quad \text { for } 1=1, \ldots, L .
$$

\section{Power Allocation}

In the proposed system, all the clusters contain two users (same cluster size), then the total transmit powers are equally allocated to the clusters, i.e., $P_{1}=\cdots=P_{L}=P_{t} / L$. This approach can be said as nearly optimal since each cluster contains users with nearly similar channel gain distinctness [6].

With the increasing of the power difference between two users in a same cluster, the SIC performance of the strong can be better, and the intracluster interference of the weak user can be also reduced because the power allocated to the signal of the strong user is decreased. So we propose a power allocation scheme for intracluster power allocation which maximizes the power difference to help perform SIC and decrease the intracluster interference for the weak user, while keeping the data rates of the two users are greater than or equal to their own minimum rate requirements. The formulation is as follows:

$$
\begin{aligned}
& \alpha_{l}^{*}=\arg \max _{\alpha_{l}} \mathrm{P}_{\text {dif }}=\arg \max _{\alpha_{l}}\left[\left(1-\alpha_{l}\right)-\alpha_{l}\right] \\
& \text { subject to } \log _{2}\left(1+\frac{\left\|\mathbf{h}_{l, 1} \mathbf{F}_{\mathrm{RF}} \mathbf{w}_{l, 1}\right\|^{2} P_{l} \alpha_{l}}{\sigma^{2}}\right) \geq \widetilde{R}_{l, 1}, \\
& \log _{2}\left(1+\frac{\left\|\mathbf{h}_{l, 2} \mathbf{F}_{\mathrm{RF}} \mathbf{w}_{l, 2}\right\|^{2} P_{l}\left(1-\alpha_{l}\right)}{\left\|\mathbf{h}_{l, 2} \mathbf{F}_{\mathrm{RF}} \mathbf{w}_{l, 1}\right\|^{2} P_{l} \alpha_{l}+\sigma^{2}}\right) \geq \widetilde{R}_{l, 2},
\end{aligned}
$$

where $\mathrm{P}_{\text {dif }}$ is the power coefficient difference between the two users. Here, $\widetilde{R}_{l, 1}$ and $\widetilde{R}_{l, 2}$ are defined as the strong and the weak users' rates in the $l$-cluster if the two users would be supported by conventional TDMA hybrid beamforming system, respectively, i.e., $\widetilde{R}_{l, i}=1 / 2 R_{l, i, T D M A-B F}$. In conventional hybrid beamforming system with $L$ RF chains, $2 L$ users need two time slots to be supported, while it only takes one time slot to support $2 L$ users in MIMO-NOMA system. So $R_{l, i, T D M A-B F}$ is multiplied by $1 / 2 . R_{l, i, T D M A-B F}$ is defined as

$$
R_{l, i, T D M A-B F}=\log _{2}\left(1+\frac{\left\|\mathbf{h}_{l, i} \mathbf{B}_{i} \mathbf{f}_{l, i}^{\mathrm{BB}}\right\|^{2} P_{l}}{\sigma^{2}}\right) .
$$

where $\mathbf{B}_{i}$ is the analog beamforming matrix containing all the feedback beams of the $i$-user in each cluster. $\mathbf{f}_{l, i}^{\mathrm{BB}}$ is designed for the $i$-user in the $l$-th cluster by using ZF-BF based on the effective channels of the $i$-user in each cluster presented in [34].

For the strong user, the power allocation coefficient $\alpha_{l}$ that ensures its rate is found by solving (19). Solving (19) for $\alpha_{l}$ gives

$$
\Longrightarrow \alpha_{l} \geq \frac{\sqrt{\gamma_{l, 1}+1}-1}{\varepsilon_{l, 1}}=\alpha_{\mathrm{inf}},
$$

where $\gamma_{l, i}=\left\|\mathbf{h}_{l, i} \mathbf{B}_{i} \mathbf{f}_{l, i}^{\mathrm{BB}}\right\|^{2} P_{l} / \sigma^{2}$ and $\varepsilon_{l, 1}=\left\|\mathbf{h}_{l, 1} \mathbf{F}_{\mathrm{RF}} \mathbf{w}_{l, 1}\right\|^{2} P_{l} / \sigma^{2}$. The least value of power allocation coefficient $\alpha_{l}$ to ensure that NOMA is fair to the strong user is given by the right side of (22), and any $\alpha_{l}$ satisfying (22) will lead to (19).

Similarly, if the rate of the weak user is to be at least as good as conventional hybrid BF, then (20) leads to

$$
\alpha_{l} \leq \frac{1+\varepsilon_{l, 2}-\sqrt{1+\gamma_{l, 2}}}{\varepsilon_{l, 2}+\beta_{l, 2}\left(\sqrt{1+\gamma_{l, 2}}-1\right)}=\alpha_{\mathrm{sub}},
$$

where $\beta_{l, 2}=\left\|\mathbf{h}_{l, 2} \mathbf{F}_{\mathrm{RF}} \mathbf{w}_{l, 1}\right\|^{2} P_{l} / \sigma^{2}$ and $\varepsilon_{l, 2}=\left\|\mathbf{h}_{l, 2} \mathbf{F}_{\mathrm{RF}} \mathbf{w}_{l, 2}\right\|^{2} P_{l} /$ $\sigma^{2}$. Therefore, the greatest value of the power allocation coefficient $\alpha_{l}$ to ensure that NOMA is fair to the weak user is given by the right side of (23), and any $\alpha_{l}$ satisfying (23) will lead to (20).

If $\alpha_{\text {inf }} \leq \alpha_{\text {sub }}$ is satisfied, the NOMA power allocation region is therefore defined as $\Lambda_{\mathrm{FN}}=\left[\alpha_{\mathrm{inf}}, \alpha_{\text {sub }}\right]$. Since the objective function is monotonically decreasing function of $\alpha_{l}$, the optimal power allocation coefficient for the strong user in the $l$-cluster, $\alpha_{l}^{*}$, can be given as

$$
\alpha_{l}^{*}=\arg \max _{\alpha_{l} \in \Lambda_{\mathrm{FN}}}\left(1-2 \alpha_{l}\right)=\alpha_{\mathrm{inf}} .
$$

In the case where $\alpha_{\text {inf }}>\alpha_{\text {sub }}$, (19) and (20) cannot be satisfied simultaneously. We will set $\alpha_{l}^{*}=\alpha_{\text {sub }}$ to allocate more power to the strong user to increase the system sum rate while keeping the rate of the weak user is equal to its minimum rate requirement, because the strong user has a larger channel gain and receives no intracluster interference by SIC.

\section{Simulation Results}

This section will present the computer simulation results to evaluate the performance of the proposed MIMO-NOMA scheme. We consider a multiuser downlink mmWave MIMO 


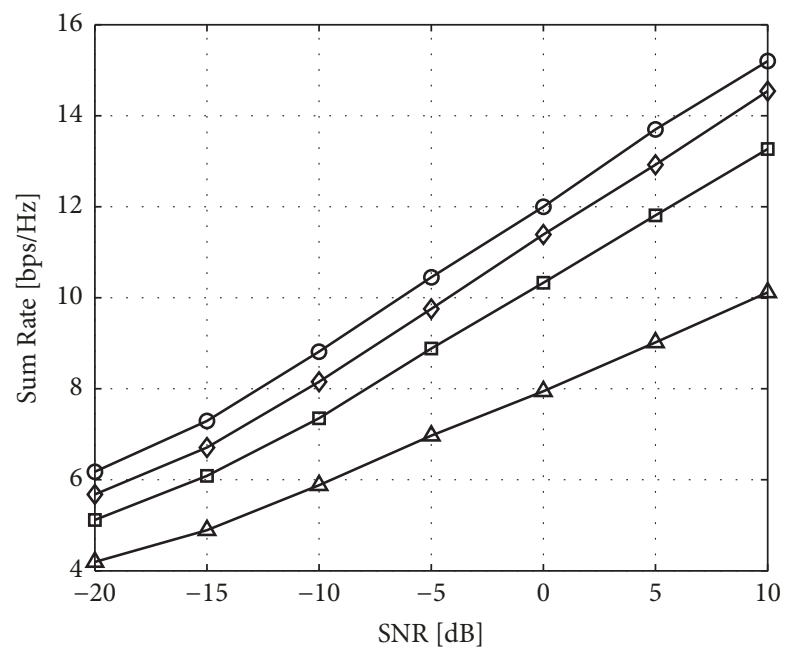

$$
\begin{aligned}
& \multimap \text { Proposed MIMO-NOMA }(\mu=0.7, \zeta=0.2) \\
& \neg \text { Proposed MIMO-NOMA }(\mu=0.3, \zeta=0.2) \\
& \square \text { Proposed MIMO-NOMA }(\mu=0.05, \zeta=0.2) \\
& \triangle \text { Conventional MIMO-OMA }(\mu=0.7, \zeta=0.2)
\end{aligned}
$$

FIGURE 1: Sum rate performances of the proposed MIMO-NOMA and conventional hybrid ZF-BF systems against $\mathrm{SNR}(\mu=0.7,0.3$, and $0.05 ; \zeta=0.2)$.

system with a BS employing a ULA of $N_{\mathrm{TX}}=100$ transmitting antennas and $N_{\mathrm{RF}}=3 \mathrm{RF}$ chains, i.e., 3 clusters in the MIMO-NOMA system. The mmWave bandwidth of the system is assumed to be $2 \mathrm{GHz}$, and its carrier frequency is assumed to be $60 \mathrm{GHz}$. The number of users $K=50$. And the all $K$ users are randomly distributed in a cell with a radius of $20 \mathrm{~m}$. We assume that there is a LoS path and 2 NLoS paths $(\mathrm{P}=2)$ for all users' channels. We set the channel parameters of each user as follows: (1) $\eta_{L O S}=2, \eta_{N L O S}=3$; (2) the normalized direction $\theta_{l, i}^{f}$ for $f=0,1,2$ follows the uniform distribution over $[-1,1]$. The beamforming codebook and RVQ codebook are quantized with $B_{\mathrm{RF}}=5$ bits and $B_{E C}=13$ bits, respectively.

Figure 1 shows the sum rate performances of the proposed MIMO-NOMA system and the conventional TDMA hybrid beamforming system mentioned in the Section 5. The key observation from Figure 1 is that the proposed MIMO-NOMA system outperforms conventional MIMOOMA system when the beam correlation and intercluster correlation thresholds are set as $\mu=0.7$ and $\zeta=0.2$, respectively. Moreover, by comparing the three cases, i.e., $\mu=$ $0.7,0.3$, and 0.05 , Figure 1 shows an improvement of sum rate of the MIMO-NOMA system as $\mu$ increases. This is because, with the increase of the beam correlation threshold $\mu$, more highly correlated users are clustered through the proposed clustering algorithm. The MIMO-NOMA system with a higher channel correlation between the users can help reduce the intercluster interference for the users by user clustering and analog beamforming.

Figure 2 shows the sum rate performances of the proposed MIMO-NOMA system, where $\mu=0.3$. Observe all the curves; we can see that the sum rate of the proposed

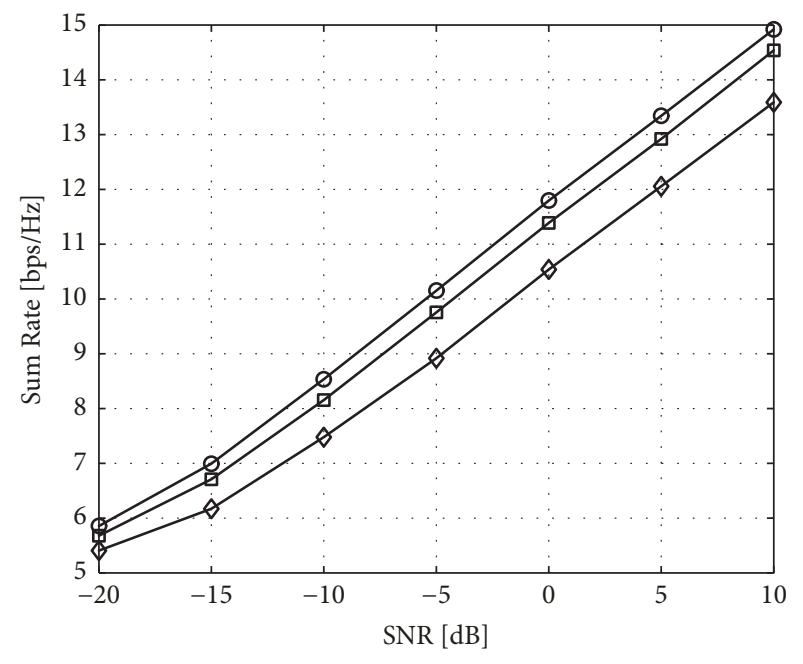

$$
\begin{aligned}
& \multimap \text { Proposed MIMO-NOMA }(\mu=0.3, \zeta=0.05) \\
& \square \text { Proposed MIMO-NOMA }(\mu=0.3, \zeta=0.2) \\
& \neg \text { Proposed MIMO-NOMA }(\mu=0.3, \zeta=0.45)
\end{aligned}
$$

FIGURE 2: Sum rate performances of the proposed MIMO-NOMA system against $\operatorname{SNR}(\mu=0.3 ; \zeta=0.05,0.2$, and 0.45$)$.

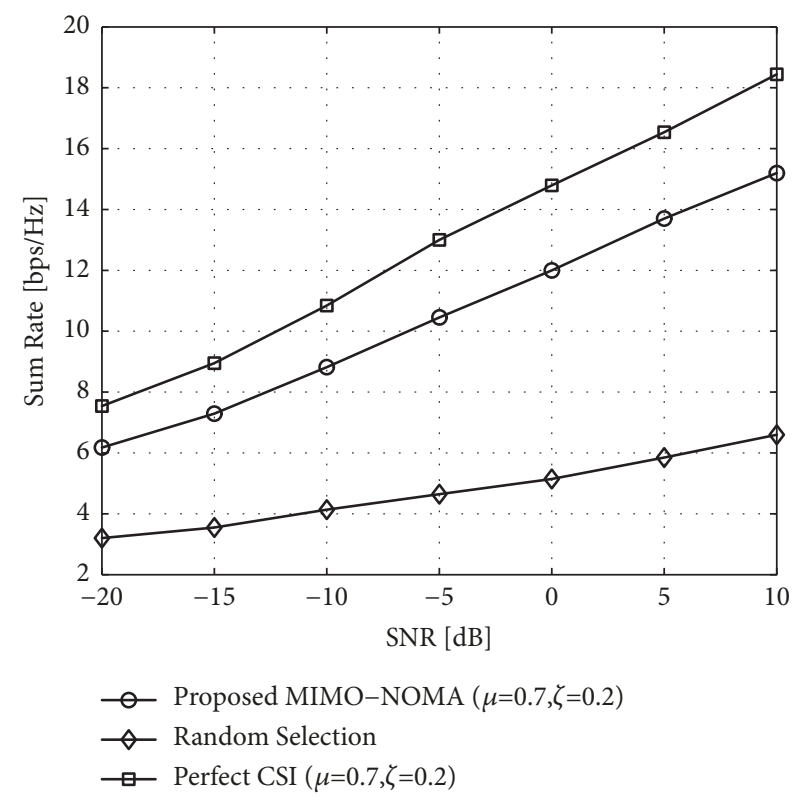

FIGURE 3: Comparison of sum rate performance between different schemes for MIMO-NOMA system against $\operatorname{SNR}(\mu=0.7 ; \zeta=0.2)$.

system increases as the intercluster correlation threshold $\zeta$ decreases. These results are expected because when $\zeta$ is reduced, clusters having lower intercluster correlations can be selected per the proposed clustering algorithm. A lower correlation between the selected clusters can help reduce the intercluster interference for the users.

Figure 3 shows the sum rate performances in three cases: randomly selecting, perfect CSI, and the proposed scheme. Randomly selecting means selecting two users from all the $K$ users as a cluster randomly. Perfect CSI means that perfect 


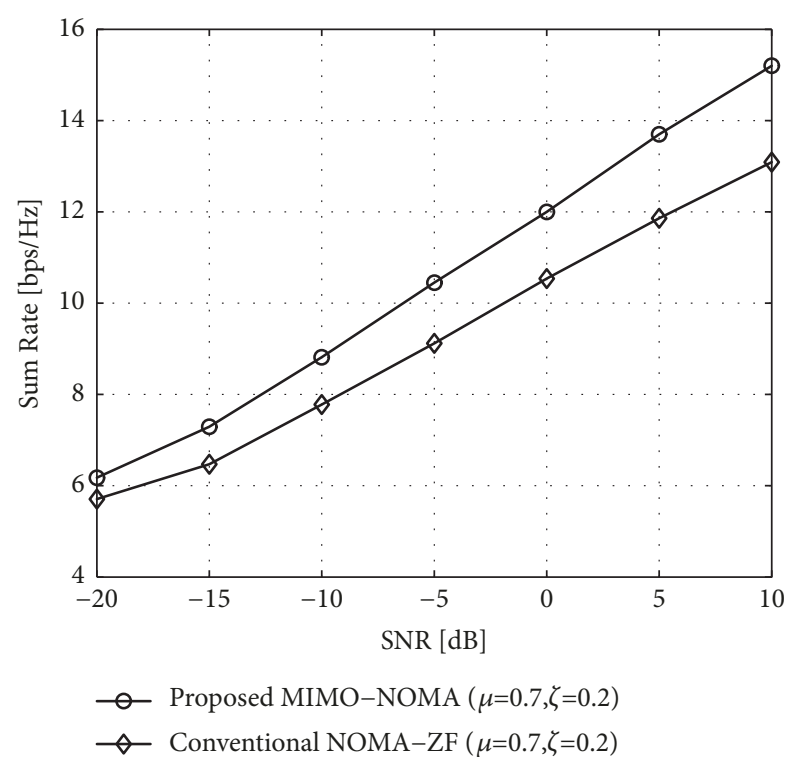

FIGURE 4: Sum rate performances of the proposed hybrid beamforming and conventional NOMA-ZF against SNR $(\mu=0.7 ; \zeta=0.2)$.

CSI is assumed to be available at BS to generate beamforming matrices and cluster users with their channel correlation and channel gains instead of beam correlation and CQI values by Algorithm 1. Randomly selecting is the simplest of the three cases, but results in a degraded sum rate performance because this scheme randomly clusters users ignoring their intercluster interferences. On the other hand, the results show that the sum rate of the proposed scheme is lower than that of perfect CSI but requires less feedback overhead.

Next, we examine the advantage of the proposed hybrid beamforming for the MIMO-NOMA system with the conventional NOMA-ZF, which is performed by utilizing ZF$\mathrm{BF}$ based on the effective channel information of the strong user of each cluster [12], cf. Figure 4. At very low SNR, the two beamforming schemes asymptotically approach the same sum rate. As SNR increases, the proposed beamforming scheme achieves a larger performance gain. This is because the proposed beamforming scheme reduces the interference of the weak users and the conventional NOMA-ZF does not.

Finally, we compare the proposed mmWave massive MIMO-NOMA scheme with limited feedback with the random beamforming scheme with one-bit feedback in [16]. The central angle of the sector is set as $2 \Delta=0.2$, and the power allocation coefficients of the two users in each cluster are set as $1 / 4$ and $3 / 4$. The number of the sectors in the random beamforming scheme is set as 3 , which is the same as the number of RF chains in the proposed scheme. As can be observed from Figure 5, the performance of the proposed scheme degrades with decreasing the number of quantization bit $B_{E C}$. The figure also shows that the feedback overhead of the proposed scheme is larger than that of the random beamforming scheme with one-bit feedback, but it achieves better performance.

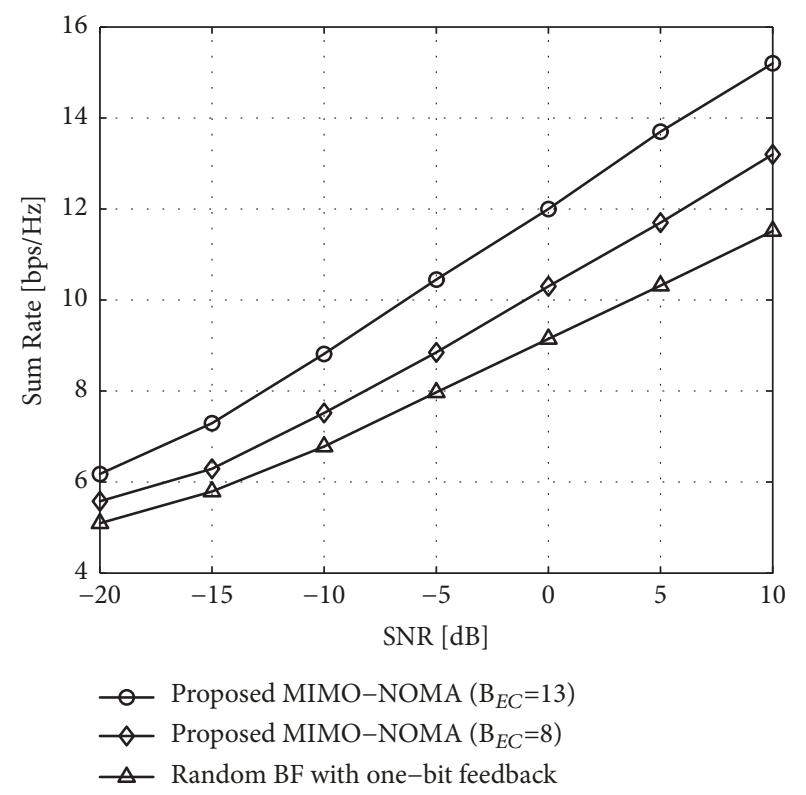

FIGURE 5: Sum rate performances of the proposed schemes with different quantization bits $(\mu=0.7 ; \zeta=0.2)$ and the random beamforming scheme with one-bit feedback $(\Delta=0.1)$ against SNR.

\section{Conclusions}

In this paper, we propose a novel mmWave MIMO-NOMA system with limited feedback. A low-complexity user clustering algorithm and hybrid beamforming scheme have been designed with the limited feedback CSI. On this basis, we propose a power allocation solution to maximize the power difference between the two users in each cluster under the rate constraints of the two users. Simulation results show that the proposed MIMO-NOMA scheme achieves a better sum rate performance than conventional MIMO-OMA, and the interand intracluster interferences and feedback overhead are reduced by applying the proposed clustering, beamforming, and effective power allocation algorithms. Therefore, the proposed scheme represents a feasible option to ensure that the mmWave MIMO-NOMA system provides an improved sum rate performance with limited feedback.

\section{Data Availability}

All data included in this study are available upon request by contact with the corresponding author.

\section{Conflicts of Interest}

The authors declare that there are no conflicts of interest regarding the publication of this paper.

\section{Acknowledgments}

This paper was supported by National Natural Science Foundation of China under Grant 61871321, National Science and Technology Major Project under Grant 2016ZX03001016, and 
Innovation Team Project of Shaanxi Province under Grant 2017KCT-30-02.

\section{References}

[1] M. Vaezi, Z. Ding, and H. V. Poor, Multiple Access Techniques for $5 G$ Wireless Networks and Beyond, Springer International Publishing, 2019.

[2] Z. Ding, X. Lei, G. K. Karagiannidis, R. Schober, J. Yuan, and V. K. Bhargava, "A survey on non-orthogonal multiple access for $5 \mathrm{~g}$ networks: research challenges and future trends," IEEE Journal on Selected Areas in Communications, vol. 35, no. 10, pp. 2181-2195, 2017.

[3] S. M. R. Islam, N. Avazov, O. A. Dobre, and K.-S. Kwak, "Power-domain non-orthogonal multiple access (NOMA) in $5 \mathrm{G}$ systems: potentials and challenges," IEEE Communications Surveys \& Tutorials, vol. 19, no. 2, pp. 721-742, 2017.

[4] B. Kimy, S. Lim, H. Kim et al., "Non-orthogonal multiple access in a downlink multiuser beamforming system," in Proceedings of the IEEE Military Communications Conference, MILCOM '13, pp. 1278-1283, San Diego, Calif, USA, 2013.

[5] X. Chen, A. Benjebbour, Y. Lan, A. Li, and H. Jiang, "Impact of rank optimization on downlink non-orthogonal multiple access (NOMA) with SU-MIMO," in Proceedings of the IEEE International Conference on Communication Systems, IEEE ICCS '14, pp. 233-237, Macau, China, 2014.

[6] M. S. Ali, H. Tabassum, and E. Hossain, "dynamic user clustering and power allocation for uplink and downlink nonorthogonal multiple access (NOMA) systems," IEEE Access, vol. 4, pp. 6325-6343, 2016.

[7] M. S. Ali, E. Hossain, and D. I. Kim, "Non-orthogonal multiple access (NOMA) for downlink multiuser MIMO systems: user clustering, beamforming, and power allocation," IEEE Access, vol. 5, pp. 565-577, 2017.

[8] M. Zeng, A. Yadav, O. A. Dobre, G. I. Tsiropoulos, and H. V. Poor, "Capacity comparison between MIMO-NOMA and MIMO-OMA with multiple users in a cluster," IEEE Journal on Selected Areas in Communications, vol. 35, no. 10, pp. 2413-2424, 2017.

[9] T. Rappaport, S. Sun, R. Mayzus et al., "Millimeter wave mobile communications for 5G cellular: it will work!", IEEE Access, vol. 1, pp. 335-349, 2013.

[10] T. S. Rappaport, R. W. Heath Jr., R. C. Daniels, and J. N. Murdock, Millimeter Wave Wireless Communications, Pearson Education, 2014.

[11] B. Wang, L. Dai, Z. Wang, N. Ge, and S. Zhou, "Spectrum and energy-efficient beamspace MIMO-NOMA for millimeterwave communications using lens antenna array," IEEE Journal on Selected Areas in Communications, vol. 35, no. 10, pp. 23702382, 2017.

[12] W. Hao, M. Zeng, Z. Chu, and S. Yang, "Energy-efficient power allocation in millimeter wave massive MIMO with nonorthogonal multiple access," IEEE Wireless Communications Letters, vol. 6, no. 6, pp. 782-785, 2017.

[13] Z. Xiao, L. Zhu, J. Choi, P. Xia, and X.-G. Xia, "Joint power allocation and beamforming for non-orthogonal multiple access (NOMA) in 5G millimeter-wave communications," IEEE Transactions on Wireless Communications, vol. 17, no. 5, pp. 29612974, 2018.

[14] M. A. Almasi, M. Vaezi, and H. Mehrpouyan, "Impact of beam misalignment on hybrid beamforming NOMA for mmWave communications," IEEE Transactions on Communications, 2018, https://arxiv.org/pdf/1808.00591.pdf.

[15] M. Dai and B. Clerckx, "Multiuser millimeter wave beamforming strategies with quantized and statistical CSIT," IEEE Transactions on Wireless Communications, vol. 16, no. 11, pp. 7025-7038, 2017.

[16] Z. Ding, P. Fan, and H. V. Poor, "Random beamforming in millimeter-wave noma networks," IEEE Access, vol. 5, pp. 76677681, 2017.

[17] Z. Ding and H. V. Poor, "Design of massive-MIMO-NOMA with limited feedback," IEEE Signal Processing Letters, vol. 23, no. 5, pp. 629-633, 2016.

[18] Z. Yang, Z. Ding, P. Fan, and G. K. Karagiannidis, "On the performance of non-orthogonal multiple access systems with partial channel information," IEEE Transactions on Communications, vol. 64, no. 2, pp. 654-667, 2016.

[19] P. Xu and K. Cumanan, "Optimal power allocation scheme for non-orthogonal multiple access with $\alpha$-fairness," IEEE Journal on Selected Areas in Communications, vol. 35, no. 10, pp. 23572369, 2017.

[20] P. Xu, Y. Yuan, Z. Ding, X. Dai, and R. Schober, "On the outage performance of non-orthogonal multiple access with 1bit feedback," IEEE Transactions on Wireless Communications, vol. 15, no. 10, pp. 6716-6730, 2016.

[21] Q. Yang, H.-M. Wang, D. W. K. Ng, and M. H. Lee, "NOMA in downlink SDMA with limited feedback: Performance analysis and optimization," IEEE Journal on Selected Areas in Communications, vol. 15, no. 10, pp. 6716-6730, 2016.

[22] "Study on Downlink Multiuser Superposition Transmission for LTE, 3rd Generation Partnership Project, 3GPP, Document RP250456, 2015".

[23] M. N. Kulkarni, A. Ghosh, and J. G. Andrews, "A comparison of MIMO techniques in downlink millimeter wave cellular networks with hybrid beamforming," IEEE Transactions on Communications, vol. 64, no. 5, pp. 1952-1967, 2016.

[24] G. Lee, Y. Sung, and J. Seo, "Randomly-directional beamforming in millimeter-wave multiuser miso downlink," IEEE Transactions on Wireless Communications, vol. 15, no. 2, pp. 1086-1100, 2016.

[25] X. Gao, L. Dai, S. Han, I. Chih-Lin, and R. W. Heath, "Energyefficient hybrid analog and digital precoding for mmwave MIMO systems with large antenna arrays," IEEE Journal on Selected Areas in Communications, vol. 34, no. 4, pp. 998-1009, 2016.

[26] X. Gao, L. Dai, Z. Chen, Z. Wang, and Z. Zhang, "Nearoptimal beam selection for beamspace mmwave massive mimo systems," IEEE Communications Letters, vol. 20, no. 5, pp. 10541057, 2016.

[27] J. Choi, "Beam selection in mm-wave multiuser MIMO systems using compressive sensing," IEEE Transactions on Communications, vol. 63, no. 8, pp. 2936-2947, 2015.

[28] T. S. Rappaport, E. Ben-Dor, J. N. Murdock, and Y. Qiao, “38 $\mathrm{GHz}$ and $60 \mathrm{GHz}$ angle-dependent propagation for cellular \& peer-to-peer wireless communications," in Proceedings of the IEEE International Conference on Communications (ICC '12), pp. 4568-4573, Ottawa, ON, Canada, 2012.

[29] M. Cordina and C. J. Debono, "A support vector machine based sub-band CQI feedback compression scheme for 3GPP LTE systems," in Proceedings of the International Symposium on Wireless Communication Systems, ISWCS '17, pp. 325-330, Bologna, Italy, 2017. 
[30] A. Alorainy and M. J. Hossain, "Cross-layer performance of downlink dynamic cell selection with random packet scheduling and partial CQI feedback in wireless networks with cell sleeping," IEEE Transactions on Wireless Communications, vol. 16, no. 8, pp. 5353-5369, 2017.

[31] O. E. Ayach, S. Rajagopal, S. Abu-Surra, Z. Pi, and R. W. Heath, "Spatially sparse precoding in millimeter wave MIMO systems," IEEE Transactions on Wireless Communications, vol. 13, no. 3, pp. 1499-1513, 2014.

[32] A. Kammoun, A. Müller, E. Björnson, and M. Debbah, "Linear precoding based on polynomial expansion: large-scale multicell mimo systems," IEEE Journal of Selected Topics in Signal Processing, vol. 8, no. 5, pp. 861-875, 2014.

[33] L. D. Nguyen, T. Q. Duong, H. Q. Ngo, and K. Tourki, "Energy efficiency in cell-free massive MIMO with zero-forcing precoding design," IEEE Communications Letters, vol. 21, no. 8, pp. 1871-1874, 2017.

[34] A. Alkhateeb, G. Leus, and R. W. Heath, "Limited feedback hybrid precoding for multi-user millimeter wave systems," IEEE Transactions on Wireless Communications, vol. 14, no. 11, pp. 6481-6494, 2015. 


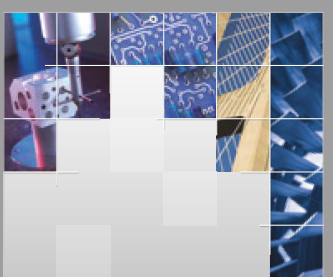

\section{Enfincering}
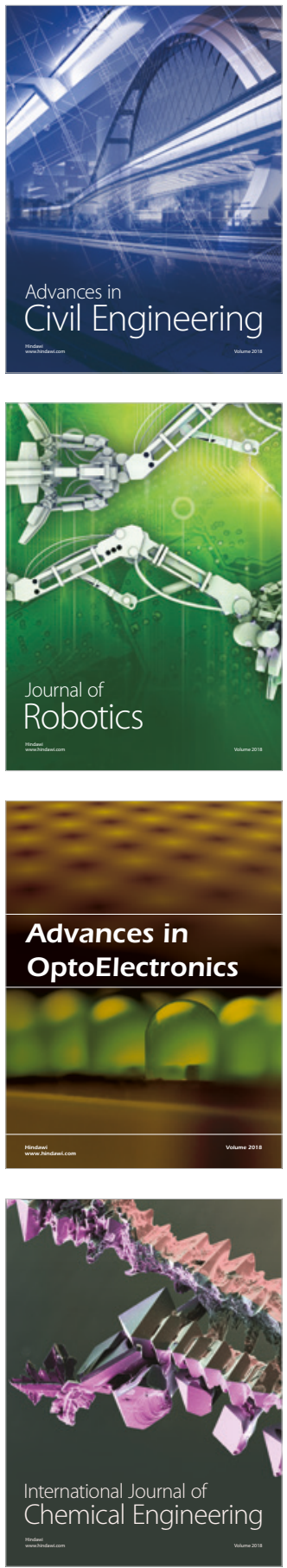

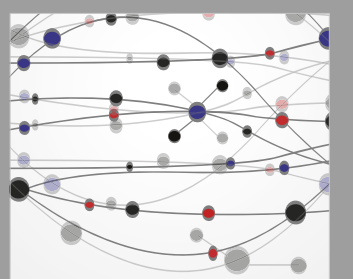

\section{Rotating \\ Machinery}

The Scientific World Journal

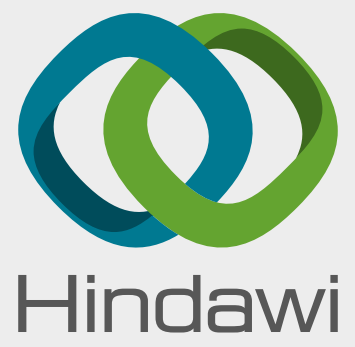

Submit your manuscripts at

www.hindawi.com
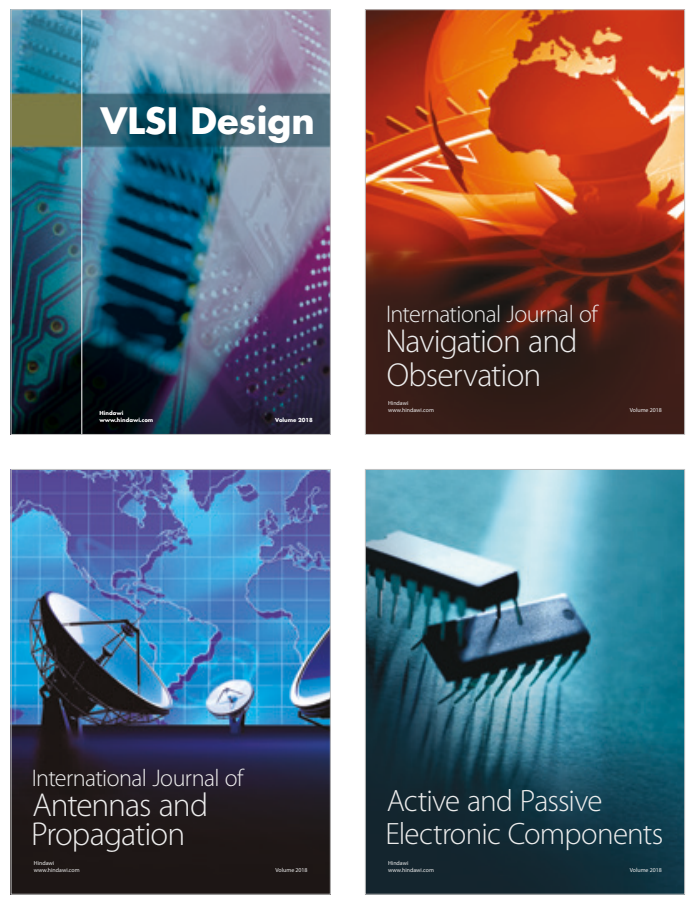
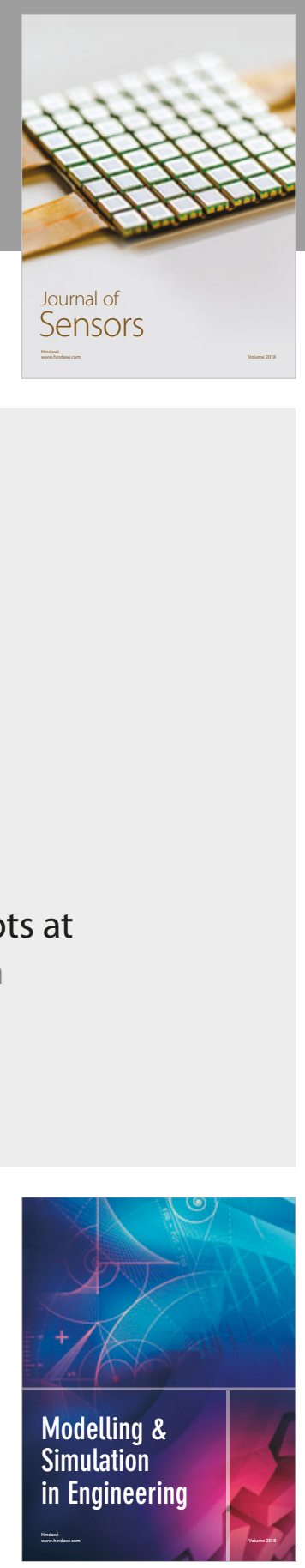

\section{Advances \\ Multimedia}
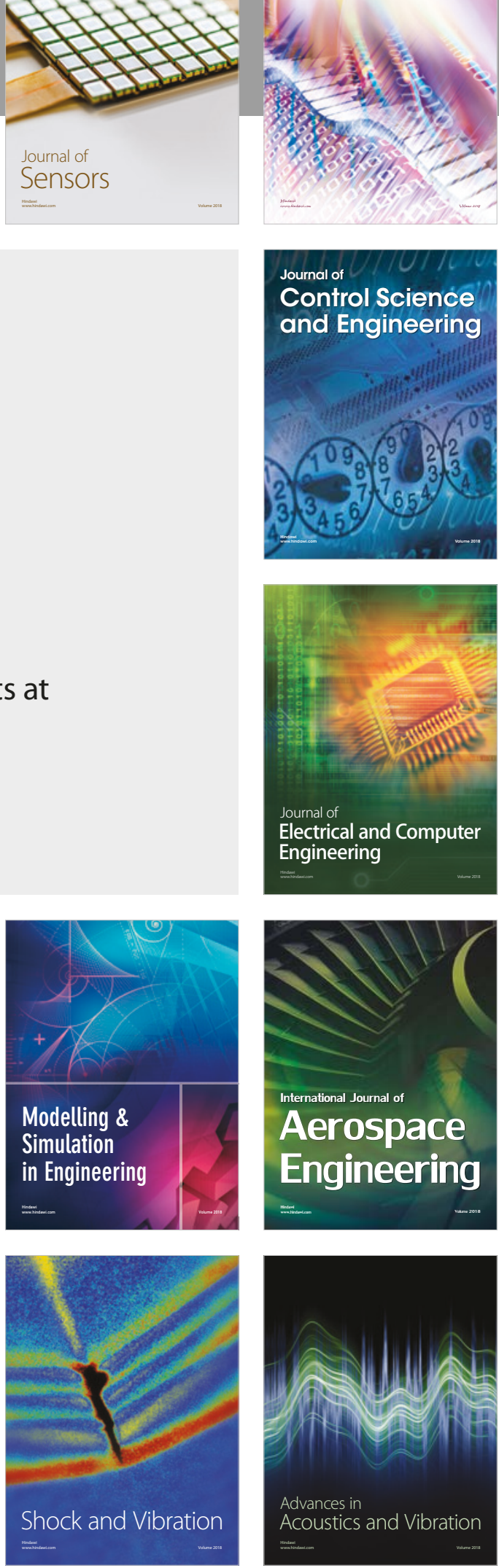